\title{
Correlates of self-reported dietary cruciferous vegetable intake and urinary isothiocyanate from two cohorts in China
}

Emily Vogtmann ${ }^{1,2,3}$, Gong Yang ${ }^{1}$, Hong-Lan Li ${ }^{3}$, Jing Wang ${ }^{3}$, Li-Hua Han ${ }^{3}$, Qi-Jun Wu ${ }^{3}$, $\mathrm{Li} \mathrm{Xie}^{3}$, Quiyin Cai ${ }^{1}$, Guo-Liang Li ${ }^{1}$, John W Waterbor ${ }^{2}$, Emily B Levitan ${ }^{2}$, Bin Zhang ${ }^{4}$, Yu-Tang Gao ${ }^{3}$, Wei Zheng ${ }^{1}$, Yong-Bing Xiang ${ }^{3}$ and Xiao-Ou Shu ${ }^{1, *}$

'Division of Epidemiology, Department of Medicine, Vanderbilt Epidemiology Center, Vanderbilt-Ingram Cancer Center, Vanderbilt University School of Medicine, 2525 West End Avenue 6th floor, Nashville, TN 37232-8300, USA: ${ }^{2}$ Department of Epidemiology, School of Public Health, University of Alabama at Birmingham, Birmingham, AL, USA: ${ }^{3}$ Department of Epidemiology and State Key Laboratory of Oncogene and Related Genes, Shanghai Cancer Institute, Renji Hospital, Shanghai Jiaotong University School of Medicine, Shanghai, People's Republic of China: ${ }^{4}$ Division of Biostatistics and Epidemiology, Cincinnati Children's Hospital Medical Center, Cincinnati, OH, USA

Submitted 23 December 2013: Final revision received 10 June 2014: Accepted 19 June 2014: First published online 7 August 2014

\begin{abstract}
Objective: To assess correlations between cruciferous vegetable intake and urinary isothiocyanate (ITC) level, in addition to glutathione $S$-transferase (GST) genotypes and other individual factors.

Design: The study included cohort participants whose urinary ITC levels had been previously ascertained. Urinary ITC was assessed using HPLC. Usual dietary intake of cruciferous vegetables was assessed using a validated FFQ and total dietary ITC intake was calculated. Recent cruciferous vegetable intake was determined. GST genotypes were assessed using duplex real-time quantitative PCR assays. Spearman correlations were calculated between the covariates and urinary ITC levels and linear regression analyses were used to calculate the mean urinary ITC excretion according to GST genotype.

Setting: Urban city in China.

Subjects: The study included 3589 women and 1015 men from the Shanghai Women's and Men's Health Studies.

Results: Median urinary ITC level was $1.61 \mathrm{nmol} / \mathrm{mg}$ creatinine. Self-reported usual cruciferous vegetable intake was weakly correlated with urinary ITC level $\left(r_{\mathrm{s}}=0.1149 ; P<0.0001\right)$, while self-reported recent intake was more strongly correlated with urinary ITC $\left(r_{\mathrm{s}}=0.2591 ; P<0.0001\right)$. Overall, the GST genotypes were not associated with urinary ITC level, but significant differences according to genotype were observed among current smokers and participants who provided an afternoon urine sample. Other factors, including previous gastrectomy or gastritis, were also related to urinary ITC level.

Conclusions: The study suggests that urinary secretion of ITC may provide additional information on cruciferous vegetable intake and that GST genotypes are related to urinary ITC level only in some subgroups.
\end{abstract}

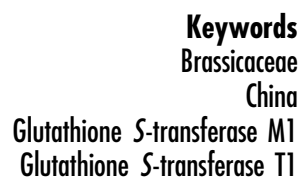

Keywords

China

Glutathione S-transferase Tl
When cruciferous vegetables are consumed, the internal glucosinolates are converted to isothiocyanate (ITC) ${ }^{(1,2)}$, a potential chemopreventive compound that inhibits phaseI enzymes (carcinogen activating) and activates phase-II enzymes (carcinogen detoxifying) ${ }^{(2)}$. Glutathione $S$-transferases (GST) are phase-II enzymes induced by ITC that catalyse conjugation of ITC for urinary excretion ${ }^{(3,4)}$ and GST gene deletions alter enzymatic GST activity ${ }^{(5,6)}$. Interactions of cruciferous vegetable consumption and/or urinary ITC levels with GST gene variants have been considered in the risk of breast, colorectum, lung and gastric cancers $^{(7-13)}$.

Cruciferous vegetable consumption is typically assessed using dietary recall such as FFQ. However, FFQ data on cruciferous vegetables have limitations, particularly recall errors $^{(14)}$. There are additional limitations when calculating dietary ITC intake values, which combine FFQ with laboratory data on the ITC content of specific vegetables. Actual ITC content varies between vegetables, so the calculated laboratory content could be different from that 
in the actual cruciferous vegetable consumed. A biomarker of cruciferous vegetable intake, like urinary ITC excretion, would not share these limitations. However, ITC and its metabolites are eliminated within $48 \mathrm{~h}$ after cruciferous vegetable consumption ${ }^{(15)}$. Therefore, urinary ITC level reflects very recent intake, whereas an FFQ assesses intake over longer periods of time.

Although intake of cruciferous vegetables and GST gene polymorphisms are likely the strongest determinants of urinary ITC excretion, factors such as age, gender, BMI and kidney function have all been associated with urinary excretion of metabolites ${ }^{(16-18)}$. Therefore, our goal was to identify factors associated with urinary ITC levels using data from the Shanghai Women's Health Study (SWHS) and the Shanghai Men's Health Study (SMHS).

\section{Methods}

\section{Source population}

The SWHS and SMHS are prospective, population-based cohort studies in Shanghai, China ${ }^{(19,20)}$. In brief, for the SWHS, 74941 women living in Shanghai aged $40-70$ years were recruited from 1996 to 2000. For the SMHS, 61483 men aged 40-74 years were recruited from 2002 to 2006. Participation rates for the SWHS and SMHS were $92.7 \%$ and $74.1 \%$, respectively. Trained interviewers administered surveys and obtained anthropometric measurements and biological samples (spot urine, blood and/or buccal cells). Both studies were conducted according to the guidelines laid down in the Declaration of Helsinki and all procedures involving human subjects were approved by the Institutional Review Boards at the Shanghai Cancer Institute and Vanderbilt University Medical Center. Written informed consent was obtained from all participants.

\section{Nested case-control study participants}

Data included in the current study were drawn from three nested SWHS case-control studies and one SMHS study that were conducted to assess the association between urinary ITC and cancer. All urine samples were collected prior to diagnosis of any cancer. The methods for the colorectal cancer and lung cancer studies in the SWHS have been published $^{(9,10)}$. From the SWHS, 430 breast cancer cases diagnosed before February 2007, 328 colorectal cancer cases diagnosed before January 2006 and 232 lung cancer cases diagnosed before February 2003 were selected. From the SMHS, 341 colorectal cancer cases diagnosed before January 2011 were selected. In both cohorts, incidence density sampling was used to select controls that were free of cancer at the time of the case's cancer diagnosis. Since controls could have developed cancer after selection we considered participants who developed cancer (up to 31 December 2010) to be cases and all others to be non-cases. We combined data from all four nested case-control studies with 3603 women and 1020 men.

\section{Assessment of cruciferous vegetable and dietary isothiocyanate intakes}

Usual dietary intake over the past 12 months was assessed at baseline. The FFQ have good validity and reliability ${ }^{(21,22)}$ and assessed intake of common cruciferous vegetables, including Chinese greens, green cabbage, Chinese cabbage/ bok choy cabbage, cauliflower and white turnip/radish. Average intake of dietary ITC was derived by multiplying the reported amount of consumption of each cruciferous vegetable by the ITC content in Asia ${ }^{(23,24)}$. At baseline urine sample collection, participants were asked about recent cruciferous vegetable intake including the total number of times they consumed green cabbage, Chinese cabbage and cauliflower during the past week and past $24 \mathrm{~h}$, and how many days or hours before sample collection they last consumed cruciferous vegetables.

\section{Measurement of urinary isotbiocyanate}

Pre-diagnosis urine samples were measured for ITC level in all four studies following an identical laboratory protocol. HPLC was used to determine total urinary ITC as previously described ${ }^{(10,25)}$. Laboratory staff were blinded to the samples' case-control status and all urine samples and standards were assayed in triplicate for the SWHS and duplicate for the SMHS. In each laboratory run, representative standards and a reagent blank were included. A standard curve was created weekly using data from samples of $\mathrm{N}$-acetyl-L-cysteine conjugates of phenethyl ITC $(0 \cdot 2-25 \mathrm{mmol} / \mathrm{l})$ in urine from individuals on a controlled diet. The average of the ITC measurements for each participant was used for analysis. If the standard deviation of the mean was greater than $10 \%$, the sample was reanalysed. The limit of detection for urinary ITC was $0 \cdot 1 \mu \mathrm{mol} / \mathrm{l}$. For undetectable ITC levels ( $n 350$ for SWHS; $n 8$ for SMHS), the value was set to $0 \cdot 1 \mu \mathrm{mol} / \mathrm{l}$ divided by the square root of two. Urinary creatinine was measured using the Jaffé alkaline picrate procedure $^{(26)}$. All ITC levels were adjusted for urine creatinine level and reported as $\mathrm{nmol} / \mathrm{mg}$ creatinine.

For the SWHS, urinary ITC analysis was conducted in three batches. Batch 1 was completed in July 2006, batch 2 in August 2007 and batch 3 in August 2008. The SMHS analysis was completed in August 2012. Time between sample collection and sample processing was adjusted in the analysis to account for storage time. The within-batch and betweenbatch CV were $15.1 \%$ and $13.7 \%$, respectively.

\section{GST genotyping}

DNA was extracted from blood (86.1\%) and buccal cell (13.9\%) samples. For both the SWHS and SMHS, copy numbers (0, 1 or 2 gene copies) of the GSTM1 and GSTT1 genes were assessed using duplex real-time quantitative PCR-based assays using methods previously described ${ }^{(27)}$. The sequences used in assay design were obtained from GenBank (GSTM1, NM_000561 and GSTT1, NM_000853). Real-time PCR were conducted in a 384-well plate in an ABI PRISM 7900 Sequence Detection System (Applied 
Biosystems, Foster City, CA, USA). Laboratory staff were blinded to the samples' case-control status. Coriell DNA samples containing 0, 1 or 2 copies of the GSTM1 and GSTT1 genes were included for internal quality control. The concordance rate for quality control samples, including water, Coriell DNA and blinded DNA samples was $100 \%$. GSTM1 and GSTT1 genotypes were within Hardy-Weinberg equilibrium among the non-cases from the SWHS $(P=0.1143$ and $P=0.6924$ for GSTM1 and GSTT1, respectively) and SMHS $(P=0.1897$ and 0.8361 for GSTM1 and GSTT1, respectively).

\section{Other covariates of interest}

Additional variables available for study included age and education, dietary, behavioural and medical factors assessed at baseline. Participants with missing data for education (four women and nineteen men) were set as the most common category, high-school education. BMI was calculated from the interviewer-measured height and weight. Behavioural characteristics included cigarette smoking, alcohol consumption, tea consumption, ginseng intake, amount of exercise per day $(\mathrm{MET} \times \mathrm{h} / \mathrm{d}$, where $\mathrm{MET}=$ metabolic equivalent of task) and menopausal status for women. The two women with missing menopausal status were classified as premenopausal because they were younger than the median age of menopause ( 49.5 years). Self-reported prevalent conditions (pulmonary tuberculosis, chronic bronchitis, asthma, chronic gastritis, chronic hepatitis, gallstones, diabetes, high blood pressure, CHD, stroke and polyps) as well as previous surgical interventions (gastrectomy and cholecystectomy) were considered. Because urinary ITC levels may be altered by diminished kidney function, we categorized participants as having a history of chronic kidney disease (International Classification of Diseases, ninth revision 9 (ICD-9) code: 403, 404 and 585; $n$ 3), nephritis (ICD-9 code: 580-589; $n$ 31), other urinary disorders (ICD-9 code: 590-599; $n$ 148) or 'any urinary disorder' (ICD-9 code: 403, 404 and 580-599; $n$ 177), and assessed the effect of 'any urinary disorder' in stratified analyses.

\section{Statistical analysis}

We excluded four women who had cancer prior to baseline, four with extreme reported total energy intake $(<2092$ or $>14644 \mathrm{~kJ} / \mathrm{d}(<500$ or $>3500 \mathrm{kcal} / \mathrm{d}))$, five with missing data for both GST genes and one woman with missing BMI data. We excluded one man with extreme reported total energy intake $(<2092$ or $>17593 \mathrm{~kJ} / \mathrm{d}(<500$ or $>4200 \mathrm{kcal} / \mathrm{d}))$ and four with missing data on both GST genes. After these exclusions, 3589 women (1071 cases and 2518 non-cases) and 1015 men (350 cases and 665 non-cases) remained for analysis.

Descriptive statistics were calculated for the SWHS and SMHS participants. Spearman correlations $\left(r_{\mathrm{s}}\right)$ were calculated between covariates and urinary ITC and adjusted for sex and batch effects. Spearman correlations of usual cruciferous vegetable and dietary ITC intakes and recent cruciferous vegetable intake with urinary ITC levels were calculated adjusted for batch, sex and total energy intake.

Linear regression analysis was applied to evaluate the association between the GST gene variants and smoking history with urinary ITC levels. Urinary ITC was natural log transformed to approximate normality and the $\beta$ estimate and $95 \%$ confidence intervals were back-transformed to the original scale for presentation. Since smoking is a GST inducer and since participants who provided urine samples in the afternoon would have been more likely to have recently consumed cruciferous vegetables, we evaluated effect modification of the association between GST gene variants and urinary ITC by smoking status and morning $v$. afternoon urine sample collection using stratified linear regression models. A linear prediction model of the natural $\log$ of ITC was created using backwards selection. Variables with a $P$ value $<0 \cdot 10$ remained in the model. Since it is possible the individuals who developed a cancer over followup may have had subclinical diseases or conditions at study enrolment that may affect dietary intake and analyses of the study, we conducted additional analyses restricted to those cancer-free individuals. Similar association patterns were observed. Therefore, results from the analyses of all samples were reported. The SAS $9 \cdot 3$ statistical software package was used for all analyses and a two-sided $P$ value $<0.05$ was considered statistically significant.

\section{Results}

Women and men from the SWHS and SMHS differed on several baseline characteristics including age, education, smoking history, alcohol and tea consumption, family history of cancer, BMI and leisure-time physical activity. The participants reported different intakes of cruciferous vegetables with a median of $82.5 \mathrm{~g} / \mathrm{d}$ for women and $90.9 \mathrm{~g} / \mathrm{d}$ for men. However, urinary ITC levels were similar for women and men with a median of 1.7 and $1.5 \mathrm{nmol} /$ mg creatinine, respectively (Table 1 ). Urinary ITC levels ranged from undetectable to $602.6 \mathrm{nmol} / \mathrm{mg}$ creatinine, with a median of $1.61 \mathrm{nmol} / \mathrm{mg}$ creatinine.

No strong correlations were observed between baseline sociodemographic, behavioural or physical characteristics and urinary ITC. Similarly, baseline prevalent conditions and prior surgeries were generally uncorrelated with urinary ITC. And no strong correlations were detected between the matching variables and urinary ITC levels (see online supplementary material, Supplemental Table 1).

Urinary ITC was significantly, although weakly, correlated with baseline usual consumption of cruciferous vegetables $\left(r_{\mathrm{s}}=0.1149, P<0.0001\right)$ and dietary ITC $\left(r_{\mathrm{s}}=0.1172\right.$, $P<0.0001)$. The correlations for total cruciferous vegetables appeared to be stronger in men $\left(r_{\mathrm{s}}=0.1733\right)$ than women $\left(r_{\mathrm{s}}=0.0988\right)$. However, no interaction was observed between cruciferous vegetable intake and $\operatorname{sex}(P=0.7845)$ or between cruciferous vegetable intake and batch $(P=0.3897)$ 
Table 1 Demographic characteristics of the included participants from the Shanghai Women's and Men's Health Studies

\begin{tabular}{|c|c|c|c|c|}
\hline & \multicolumn{2}{|c|}{ SWHS ( $n$ 3589) } & \multicolumn{2}{|c|}{ SHMS (n 1015) } \\
\hline & Median or $\%$ & IQR & Median or $\%$ & IQR \\
\hline Age (years) & 59.5 & $49 \cdot 1-65 \cdot 6$ & $65 \cdot 6$ & $56 \cdot 2-70 \cdot 6$ \\
\hline \multicolumn{5}{|l|}{ Educational level (\%) } \\
\hline$\leq$ Elementary school & $35 \cdot 7$ & - & $14 \cdot 0$ & - \\
\hline Middle school & 29.8 & - & $32 \cdot 3$ & - \\
\hline High school & $22 \cdot 8$ & - & $27 \cdot 0$ & - \\
\hline$\geq$ Coollege & $11 \cdot 8$ & - & $26 \cdot 7$ & - \\
\hline \multicolumn{5}{|l|}{ Cigarette smoking (\%) } \\
\hline Never & $96 \cdot 6$ & - & 38.4 & - \\
\hline Past & 0.6 & - & $18 \cdot 2$ & - \\
\hline Current & 2.9 & - & 43.4 & - \\
\hline \multicolumn{5}{|l|}{ Alcohol consumption (\%) } \\
\hline Never & $97 \cdot 3$ & - & 68.4 & - \\
\hline Ever & $2 \cdot 7$ & - & 31.6 & - \\
\hline \multicolumn{5}{|l|}{ Tea consumption (\%) } \\
\hline Never & 74.3 & - & $37 \cdot 8$ & - \\
\hline Ever & $25 \cdot 7$ & - & $62 \cdot 2$ & - \\
\hline \multicolumn{5}{|l|}{ Ginseng use (\%) } \\
\hline Never & $66 \cdot 1$ & - & 63.7 & - \\
\hline Ever & 33.9 & - & $36 \cdot 3$ & - \\
\hline \multicolumn{5}{|l|}{ Family history of cancer (\%) } \\
\hline Yes & $26 \cdot 8$ & - & 31.9 & - \\
\hline No & 73.2 & - & 68.1 & - \\
\hline BMl $\left(\mathrm{kg} / \mathrm{m}^{2}\right)$ & $24 \cdot 3$ & $22 \cdot 1-26 \cdot 7$ & 23.9 & $21 \cdot 8-26 \cdot 1$ \\
\hline Leisure-time physical activity $(\mathrm{MET} \times \mathrm{h} / \mathrm{d})$ & 0.0 & $0.0-1.2$ & 0.3 & $0.0-2.9$ \\
\hline Total energy intake $(\mathrm{kJ} / \mathrm{d})$ & 6822 & $5816-7902$ & 7644 & $6520-8987$ \\
\hline Total energy intake $(\mathrm{kcal} / \mathrm{d})$ & $1630 \cdot 6$ & $1390 \cdot 1-1888 \cdot 6$ & $1826 \cdot 9$ & $1558 \cdot 2-2147 \cdot 9$ \\
\hline Cruciferous vegetable intake $(\mathrm{g} / \mathrm{d})$ & 82.5 & $50 \cdot 7-131 \cdot 8$ & 90.9 & $56 \cdot 6-147 \cdot 0$ \\
\hline Dietary ITC $(\mu \mathrm{mol} / \mathrm{d})$ & $7 \cdot 0$ & $4.4-11 \cdot 2$ & $7 \cdot 9$ & $4 \cdot 7-13 \cdot 0$ \\
\hline Urinary ITC (nmol/mg creatinine) & $1 \cdot 7$ & $0.7-4.0$ & 1.5 & $0.7-3.8$ \\
\hline
\end{tabular}

SWHS, Shanghai Women's Health Study; SMHS, Shanghai Men's Health Study; IQR, interquartile range; MET, metabolic equivalent of task; ITC, isothiocyanate.

Median and IQR are presented for continuous variables.

for associations with urinary ITC. Stronger correlations were observed for the measures of recent cruciferous vegetable intake with $r_{\mathrm{s}}=0.2591(P<0.0001)$ for the number of times cruciferous vegetables were consumed in the past week, $r_{\mathrm{s}}=0.2400(P<0.0001)$ for the number of times cruciferous vegetables were consumed in the past $24 \mathrm{~h}$ and $r_{\mathrm{s}}=-0.2877$ $(P<0.0001)$ for the number of hours since the last intake of cruciferous vegetables (Table 2 ). Usual cruciferous vegetable consumption was not strongly correlated with the measures of recent intake, with $r_{\mathrm{s}}$ of 0.0421 for the number of times cruciferous vegetables were consumed in the past week, 0.0239 for the number of times cruciferous vegetables were consumed in the past $24 \mathrm{~h}$ and -0.0240 for the number of hours since the last intake of cruciferous vegetables. Additional adjustment for other covariates, including morning or afternoon urine sample collection and smoking, did not materially alter the correlations (results not shown). When analyses were restricted to non-cases, correlations were similar (results not shown). When analyses were restricted to participants with 'no urinary disorder' the correlations were similar, but in participants with 'any urinary disorder' the correlations were attenuated; however, only 177 participants were categorized as 'any urinary disorder' (results not shown).

Mean levels of urinary ITC were lower among participants with the GST null genotypes; however, the observed differences were not statistically significant (Table 3). Adjustment for baseline covariates that were significantly associated with ITC level did not alter associations. Similarly, inclusion of self-reported usual cruciferous vegetable intake did not materially change results (results not shown). Smokers had lower urinary ITC levels, with geometric mean urinary ITC levels of 1.59 (95\% CI 1.52, 1.66), 1.29 (95\% CI $1.04,1.59)$ and $1.40(95 \%$ CI $1.22,1.61) \mathrm{nmol} / \mathrm{mg}$ creatinine for never, past and current smokers, respectively, after adjustment for batch and sex.

When the analyses were stratified by smoking status, current smokers with the carrier genotype for both the GSTM1 and GSTT1 genes had a significantly higher urinary level of ITC than the other combinations, with a geometric mean of 1.91 (95\% CI $1.52,2.42) \mathrm{nmol} / \mathrm{mg}$ creatinine. Current smokers with the carrier genotype of the GSTM1 gene also had a slightly higher urinary excretion of ITC, with a geometric mean of 1.66 (95\% CI 1.40, 1.96) nmol/mg creatinine (Table 4). When stratified by morning or afternoon urine sample collection, a difference in urinary ITC level was detected for the GSTM1 gene $(P=0.0436)$ and the combination GSTM1/GSTT1 gene category $(P=0.0328)$ among participants who provided a urine sample in the afternoon (Table 5). However, no differences in self-reported recent cruciferous vegetable consumption (past $24 \mathrm{~h}$ or week) were 
Table 2 Spearman correlations between reported dietary intakes and urinary isothiocyanate (ITC) levels among the included participants from the Shanghai Women's and Men's Health Studies

\begin{tabular}{|c|c|c|c|c|c|c|}
\hline & \multicolumn{2}{|c|}{ Women } & \multicolumn{2}{|c|}{ Men } & \multicolumn{2}{|c|}{ Women and men } \\
\hline & \multicolumn{2}{|c|}{ Model 1} & \multicolumn{2}{|c|}{ Model 1} & \multicolumn{2}{|c|}{ Model 2} \\
\hline & $r_{\mathrm{s}}$ & $P$ value & $r_{\mathrm{s}}$ & $P$ value & $r_{\mathrm{s}}$ & $P$ value \\
\hline Cruciferous vegetables $(\mathrm{g} / \mathrm{d})$ & 0.0988 & $<0.0001$ & 0.1733 & $<0.0001$ & 0.1149 & $<0.0001$ \\
\hline Greens, Chinese greens $(\mathrm{g} / \mathrm{d})$ & 0.0809 & $<0.0001$ & $0 \cdot 1320$ & $<0.0001$ & 0.0921 & $<0.0001$ \\
\hline Green cabbage $(\mathrm{g} / \mathrm{d})$ & 0.0970 & $<0.0001$ & $0 \cdot 1011$ & 0.0013 & 0.0975 & $<0.0001$ \\
\hline Chinese cabbage/bok choy cabbage (g/d) & 0.0384 & 0.0217 & 0.0682 & 0.0299 & 0.0433 & 0.0033 \\
\hline Cauliflower $(\mathrm{g} / \mathrm{d})$ & 0.0505 & 0.0025 & 0.0977 & 0.0018 & 0.0607 & $<0.0001$ \\
\hline White radish/turnip (g/d) & 0.0882 & $<0.0001$ & 0.1903 & $<0.0001$ & 0.1104 & $<0.0001$ \\
\hline Dietary ITC $(\mu \mathrm{mol} / \mathrm{d})$ & 0.1055 & $<0.0001$ & $0 \cdot 1617$ & $<0.0001$ & 0.1172 & $<0.0001$ \\
\hline Cruciferous vegetables (times/past week) & 0.2530 & $<0.0001$ & 0.2839 & $<0.0001$ & 0.2591 & $<0.0001$ \\
\hline Cruciferous vegetables (times/past $24 \mathrm{~h}$ ) & 0.2416 & $<0.0001$ & 0.2365 & $<0.0001$ & 0.2400 & $<0.0001$ \\
\hline Hours since last cruciferous vegetable intake & -0.2839 & $<0.0001$ & -0.3048 & $<0.0001$ & -0.2877 & $<0.0001$ \\
\hline
\end{tabular}

Model 1 adjusted for assay batch and total energy intake.

Model 2 adjusted for assay batch, total energy intake and sex.

Table 3 Geometric mean urinary isothiocyanate levels ( $\mathrm{nmol} / \mathrm{mg}$ creatinine) by glutathione $S$-transferase gene (GST) copy number among the included participants from the Shanghai Women's and Men's Health Studies

\begin{tabular}{lcccc}
\hline & $n$ & Mean & $95 \% \mathrm{Cl}$ & $P$ value \\
\hline GSTM1 & & & & \\
$\quad$ Null & 2666 & 1.51 & $1.43,1.59$ & 0.1209 \\
$\quad$ Carrier & 1895 & 1.61 & $1.51,1.71$ & \\
GSTT1 & & & $1.44,1.62$ & \\
$\quad$ Null & 2281 & 1.53 & $1.48,1.66$ & \\
$\quad$ Carrier & 2311 & 1.57 & $1.41,1.64$ & \\
GSTM1/GSTT1 & & & $1.43,1.60$ & \\
$\quad$ Both null & 1293 & 1.52 & $1.53,1.84$ \\
$\quad$ One null and one carrier & 2331 & 1.51 & 1.68 & \\
Both carrier & 925 & & \\
\hline
\end{tabular}

The model adjusted for assay batch and sex.

Table 4 Geometric mean urinary isothiocyanate levels ( $\mathrm{nmol} / \mathrm{mg}$ creatinine) by glutathione S-transferase gene (GST) copy number, stratified by smoking status, among the included participants from the Shanghai Women's and Men's Health Studies

\begin{tabular}{|c|c|c|c|c|c|c|c|c|c|c|c|c|}
\hline & \multicolumn{4}{|c|}{ Never smokers } & \multicolumn{4}{|c|}{ Past smokers } & \multicolumn{4}{|c|}{ Current smokers } \\
\hline & $n$ & Mean & $95 \% \mathrm{Cl}$ & $P$ value* & $n$ & Mean & $95 \% \mathrm{Cl}$ & $P$ value* & $n$ & Mean & $95 \% \mathrm{Cl}$ & $P$ value* \\
\hline \multicolumn{13}{|l|}{ GSTM1 } \\
\hline Null & 2241 & 1.54 & $1.45,1.63$ & \multirow[t]{2}{*}{0.2987} & 123 & 1.32 & $1.04,1.68$ & \multirow[t]{2}{*}{0.7802} & 302 & 1.36 & $1.18,1.58$ & \multirow[t]{2}{*}{0.0861} \\
\hline Carrier & 1600 & 1.62 & $1.51,1.73$ & & 75 & 1.39 & $1.02,1.89$ & & 220 & 1.66 & $1.40,1.96$ & \\
\hline \multicolumn{13}{|l|}{ GSTT1 } \\
\hline Null & 1931 & 1.55 & $1.45,1.65$ & \multirow[t]{2}{*}{0.4488} & 100 & 1.42 & $1.09,1.84$ & \multirow[t]{2}{*}{0.5476} & 250 & 1.44 & $1.23,1.69$ & \multirow[t]{2}{*}{0.8300} \\
\hline $\begin{array}{l}\text { Carrier } \\
\text { GSTM1/GSTT1 }\end{array}$ & 1918 & 1.60 & $1.50,1.71$ & & 103 & $1 \cdot 26$ & $0.97,1.64$ & & 290 & 1.48 & $1.28,1.71$ & \\
\hline Both null & 1105 & 1.53 & $1.41,1.67$ & \multirow{3}{*}{0.3523} & 52 & 1.26 & $0.87,1.82$ & \multirow{3}{*}{0.5243} & 136 & 1.55 & $1.25,1.92$ & \multirow{3}{*}{0.0200} \\
\hline One null and one carrier & 1949 & 1.55 & $1.46,1.65$ & & 113 & 1.43 & $1.12,1.84$ & & 269 & 1.29 & $1.11,1.51$ & \\
\hline Both carrier & 780 & 1.68 & $1.52,1.85$ & & 31 & 1.06 & $0.66,1.71$ & & 114 & 1.91 & $1.52,2.42$ & \\
\hline
\end{tabular}

All models adjusted for assay batch and sex.

${ }^{*} P$ value tests the difference in means within each genotype stratum.

observed between participants who provided morning or afternoon urine samples (results not shown).

The model to predict urinary ITC level using backwards selection to select from all covariates included previous gastrectomy, leisure-time physical activity, history of diabetes, history of chronic gastritis, ever consuming ginseng, history of high blood pressure, history of $\mathrm{CHD}$, the number of times cruciferous vegetables were consumed in the past $24 \mathrm{~h}$, morning sample collection, providing a blood sample, usual cruciferous vegetable intake, sample batch and hours since last intake of cruciferous vegetables. However, this model was able to predict only $11.7 \%\left(R^{2}=0 \cdot 117\right)$ of the variation in urinary ITC (see online supplementary material, Supplemental Table 2). 
Table 5 Geometric mean urinary isothiocyanate levels ( $\mathrm{nmol} / \mathrm{mg}$ creatinine) by glutathione $S$-transferase gene (GST) copy number, stratified by timing of the urine sample, among the included participants from the Shanghai Women's and Men's Health Studies

\begin{tabular}{|c|c|c|c|c|c|c|c|c|}
\hline & \multicolumn{4}{|c|}{ Morning sample } & \multicolumn{4}{|c|}{ Afternoon sample } \\
\hline & $n$ & Mean & $95 \% \mathrm{Cl}$ & $P$ value* & $n$ & Mean & $95 \% \mathrm{Cl}$ & $P$ value* \\
\hline \multicolumn{9}{|l|}{ GSTM1 } \\
\hline Null & 1188 & 1.42 & $1.32,1.54$ & \multirow[t]{2}{*}{0.9217} & 1478 & 1.58 & $1.47,1.70$ & \multirow[t]{2}{*}{0.0436} \\
\hline Carrier & 857 & 1.43 & $1.31,1.57$ & & 1038 & 1.77 & $1.63,1.93$ & \\
\hline \multicolumn{9}{|l|}{ GSTT1 } \\
\hline Null & 1011 & 1.43 & $1.31,1.55$ & \multirow[t]{2}{*}{0.9405} & 1270 & 1.61 & $1.49,1.74$ & \multirow[t]{2}{*}{0.4140} \\
\hline Carrier & 1045 & 1.43 & $1.32,1.56$ & & 1266 & 1.69 & $1.56,1.83$ & \\
\hline \multicolumn{9}{|l|}{ GSTM1/GSTT1 } \\
\hline Both null & 586 & 1.43 & $1.28,1.60$ & \multirow[t]{3}{*}{0.9737} & 707 & 1.61 & $1.45,1.78$ & \multirow[t]{3}{*}{0.0328} \\
\hline One null and one carrier & 1014 & 1.42 & $1.30,1.54$ & & 1317 & 1.59 & $1.48,1.72$ & \\
\hline Both carrier & 440 & 1.44 & $1.27,1.64$ & & 485 & 1.93 & $1 \cdot 70,2 \cdot 18$ & \\
\hline
\end{tabular}

All models adjusted for assay batch and sex.

${ }^{*} P$ value tests the difference in means within each genotype stratum.

\section{Discussion}

In the present study of Chinese men and women, selfreported intake of cruciferous vegetables was only weakly correlated with urinary ITC levels measured in a spot urine sample. The strongest correlations were observed between self-reported recent cruciferous vegetable intake and urinary ITC. Overall, urinary ITC did not appear to be related to GST gene polymorphisms, but when the data were stratified by smoking status, some differences by genotype were observed among current smokers. Additionally, among participants who provided an afternoon urine sample, those with the GSTM1-null genotype had lower urinary ITC excretion than the carrier genotype. A number of individual factors were associated with urinary ITC levels, but the linear prediction model was only able to explain approximately $12 \%$ of the variation.

In a previous study of postmenopausal women in the USA, a relatively weak correlation (Pearson correlation = $0 \cdot 22$ ) was observed between cruciferous vegetable intake from an FFQ and urinary dithiocarbamate, another biomarker of cruciferous vegetable intake ${ }^{(28)}$. This observed correlation was stronger than the correlation with usual intake in our study; however, the study assessed cruciferous vegetable intake only during the week prior to a cruciferous vegetable intervention and the correlation in our study for recent intake was similar to this finding. Another study among a Chinese population in Singapore noted statistically significant associations between consumption of cruciferous vegetables and urinary ITC $(P=0.0004)$ and between dietary ITC and urinary ITC ( $P=0.0003)$, but did not report the strength of the associations $^{(23)}$. The correlations observed in our study were all statistically significant $(P<0 \cdot 0001)$, but the correlations were weak. Relatively weak correlations $\left(r_{\mathrm{s}}=0 \cdot 16, P<0 \cdot 01\right)$ were observed in a population of female controls from the Shanghai Breast Cancer Study between both usual cruciferous vegetable intake and dietary ITC and urinary levels of ITC $^{(29)}$.
Feeding studies have shown that urinary ITC is a useful biomarker of dietary exposure to ITC, with a Spearman correlation of 0.93 between cruciferous vegetable dose and $24 \mathrm{~h}$ urinary output of ITC $^{(15,25)}$. These results are expected since urinary ITC has a peak excretion between 2 and $6 \mathrm{~h}$ after consuming cruciferous vegetables with low to no presence after 24 to $48 \mathrm{~h}^{(15)}$. Thus, urinary ITC levels are good markers for recent cruciferous vegetable intake. Our FFQ assessed usual intake over the past year and a single urine sample from each participant was available for our study. Since urinary ITC reflects recent intake and the FFQ only ascertained usual intake, the weak correlations were expected. Supporting this point, we found stronger correlations of urinary ITC with recent cruciferous vegetable intake, although the correlation was still relatively weak. Measurement error in cruciferous vegetable intake data derived from the FFQ could also attenuate the correlations. In general, FFQ data are prone to dietary recall errors $^{(14)}$, do not cover every cruciferous vegetable that produces ITC ${ }^{(30)}$ and do not account for variability in actual ITC exposure affected by growing conditions, cooking methods $^{(31)}$ and storage conditions ${ }^{(32)}$. We used ITC data from vegetables in Asia; however, this calculation excluded white turnip/radish because data on ITC content were not available $\mathrm{e}^{(23,24)}$. Some of the variation between cruciferous vegetable intake and urinary ITC appeared to be affected by chronic conditions, such as gastrointestinal disease, since in the linear prediction model both a history of chronic gastritis ( $n 938 ; 20.4 \%$ ) and previous gastrectomy ( $n 52 ; 1.1 \%$ ) were statistically associated with urinary ITC. Although this is an intriguing finding, all chronic condition data were collected by self-report and it is beyond the scope of the study to investigate potential mechanisms such as whether gastrointestinal disease changed urinary ITC levels or if participants with gastrointestinal disease changed intake of cruciferous vegetables.

ITC induces GST enzymes that in turn catalyse the conjugation of ITC $^{(2)}$, but previous research has been inconsistent regarding the effect of the GSTM1 and GSTT1 
genes on the urinary output of ITC. One study found that urinary ITC was higher among participants with the GSTM1 null genotype ${ }^{(33)}$, another found that urinary ITC was slightly higher among participants with the GSTT1 null genotype $^{(29)}$ and two other studies observed no difference by genotype ${ }^{(34,35)}$. We found no indication for a difference by genotype in our entire sample, but GSTM1 and the combination of GSTM1 and GSTT1 genotypes appeared to be associated with urinary ITC levels among current smokers. Smoking is a GST inducer and in our sample, urinary ITC level was lower for participants having a null genotype for GSTM1 or for both GSTM1 and GSTT1. The GSTM1 and the combination of GSTM1 and GSTT1 genotypes also were associated with urinary ITC levels for participants who provided an afternoon urine sample, suggesting that these genotypes may be relevant to ITC excretion rates when exposure levels are higher, since afternoon samples are presumably collected sooner following consumption of cruciferous vegetables than morning samples would be, although this may be a chance finding. Additional research is needed on the relationship between cruciferous vegetable consumption and GST gene variants including the effect of smoking and timing of urine sample collection.

Our study has a number of important strengths including the relatively large sample size, high response rate and the population-based study design of the parent study. Although there are limitations to the self-reported measures of cruciferous vegetable intake and dietary ITC, the FFQ had relatively high validity and reliability measures ${ }^{(21,22)}$. The urinary ITC measurement is limited because only a single, spot urine sample was available. Given that urinary ITC appears to have a peak excretion between 2 and $6 \mathrm{~h}$ after consumption of cruciferous vegetables ${ }^{(15)}$, our study is limited by relying on a spot urine sample. A $24 \mathrm{~h}$ urine sample would likely give a better estimate of recent cruciferous vegetable intake, but collection of a $24 \mathrm{~h}$ urine sample in a largescale epidemiological study would be extremely challenging and may lead to increased missing data and selection bias. Confounding due to unmeasured confounders could be an issue. For example, we did not assess other GST genes, such as GSTP1 or GSTA1, or other potential metabolizing genes on the urinary excretion of ITC. Finally, laboratory errors in the assessment of urinary ITC and the GST gene variants could occur; however, quality control procedures minimized errors and any bias would likely be non-differential.

\section{Conclusion}

In conclusion, self-reported usual intake of cruciferous vegetables was weakly correlated with urinary level of ITC while self-reported recent intake was more strongly correlated with urinary ITC, which suggests that urinary ITC measured in a spot urine sample is a better biomarker for recent cruciferous vegetable intake. GST gene variants, particularly GSTM1, may be important in ITC metabolism and excretion among current smokers and shortly after intake. Future research on urinary ITC should take into consideration the influence of genotype, smoking and upper gastrointestinal diseases.

\section{Acknowledgements}

Acknowledgements: The authors would like to thank the participants and the staff from the Shanghai Women's and Men's Health Studies. They also would like to thank Dr Hui Cai for statistical assistance and Regina Courtney for laboratory support from Vanderbilt University Medical Center. Financial support: This work was supported by funds from the National Institutes of Health (NIH; grant numbers R01 CA082729 and R37 CA070867) and the Vanderbilt Clinical and Translational Science Award from the National Center for Research Resources (grant number UL1 RR024975-01) which is now at the National Center for Advancing Translational Sciences (grant number UL1 TR000445-06). E.V. and H.-L.L. were supported by the Fogarty International Clinical Research Scholars and Fellows Program at Vanderbilt University (grant number R24 TW007988); and E.V. was supported by the Cancer Prevention and Control Training Program at the University of Alabama at Birmingham funded through the NIH (grant number 5R25 CA047888). The content is solely the responsibility of the authors and does not necessarily represent the official views of the NIH. The funders had no role in the design, analysis or writing of this article. Conflict of interest: None. Authorship: G.Y., Y.T.G., W.Z., Y.-B.X., and X.-O.S. designed the study. E.V., G.Y., H.-L.L., J.W., L.-H.H., Q.-J.W., L.X., Q.C., G.-L.L., J.W.W., E.B.L., B.Z., Y.-B.X. and X.-O.S. generated, collected, analysed and/or interpreted the data. E.V., G.Y., J.W.W., E.B.L., B.Z., Y.-B.X. and X.-O.S. drafted or revised the initial manuscript. All authors read and approved the final manuscript. Etbics of buman subject participation: The SWHS and SMHS were reviewed by the Institutional Review Boards at the Shanghai Cancer Institute and Vanderbilt University Medical Center.

\section{Supplementary material}

To view supplementary material for this article, please visit http://dx.doi.org/10.1017/S1368980014001505

\section{References}

1. Krul C, Humblot C, Philippe C et al. (2002) Metabolism of sinigrin (2-propenyl glucosinolate) by the human colonic microflora in a dynamic in vitro large-intestinal model. Carcinogenesis 23, 1009-1016.

2. Zhang Y, Yao S \& Li J (2006) Vegetable-derived isothiocyanates: anti-proliferative activity and mechanism of action. Proc Nutr Soc 65, 68-75. 
3. Zhang Y, Kolm RH, Mannervik B et al. (1995) Reversible conjugation of isothiocyanates with glutathione catalyzed by human glutathione transferases. Biochem Biophys Res Commun 206, 748-755.

4. Kolm RH, Danielson UH, Zhang Y et al. (1995) Isothiocyanates as substrates for human glutathione transferases: structure-activity studies. Biochem J 311, 453-459.

5. Seidegard J, Vorachek WR, Pero RW et al. (1988) Hereditary differences in the expression of the human glutathione transferase active on trans-stilbene oxide are due to a gene deletion. Proc Natl Acad Sci USA 85, 7293-7297.

6. Pemble S, Schroeder KR, Spencer SR et al. (1994) Human glutathione $S$-transferase theta (GSTT1): cDNA cloning and the characterization of a genetic polymorphism. Biochem J 300, 271-276.

7. Lee SA, Fowke JH, Lu W et al. (2008) Cruciferous vegetables, the GSTP1 Ile105Val genetic polymorphism, and breast cancer risk. Am J Clin Nutr 87, 753-760.

8. Epplein M, Wilkens LR, Tiirikainen M et al. (2009) Urinary isothiocyanates; glutathione $S$-transferase M1, T1, and P1 polymorphisms; and risk of colorectal cancer: the Multiethnic Cohort Study. Cancer Epidemiol Biomarkers Prev 18, 314-320.

9. Yang G, Gao YT, Shu XO et al. (2010) Isothiocyanate exposure, glutathione $S$-transferase polymorphisms, and colorectal cancer risk. Am J Clin Nutr 91, 704-711.

10. Fowke JH, Gao YT, Chow WH et al. (2011) Urinary isothiocyanate levels and lung cancer risk among non-smoking women: a prospective investigation. Lung Cancer $\mathbf{7 3}$, $18-24$.

11. Lam TK, Gallicchio L, Lindsley K et al. (2009) Cruciferous vegetable consumption and lung cancer risk: a systematic review. Cancer Epidemiol Biomarkers Prev 18, 184-195.

12. Moy KA, Yuan JM, Chung FL et al. (2009) Isothiocyanates, glutathione $S$-transferase M1 and T1 polymorphisms and gastric cancer risk: a prospective study of men in Shanghai, China. Int J Cancer 125, 2652-2659.

13. Kim MK \& Park JH (2009) Conference on 'Multidisciplinary approaches to nutritional problems'. Symposium on 'Nutrition and health'. Cruciferous vegetable intake and the risk of human cancer: epidemiological evidence. Proc Nutr Soc 68 103-110.

14. Natarajan L, Flatt SW, Sun X et al. (2006) Validity and systematic error in measuring carotenoid consumption with dietary self-report instruments. Am J Epidemiol 163, 770-778.

15. Kristensen M, Krogholm KS, Frederiksen H et al. (2007) Urinary excretion of total isothiocyanates from cruciferous vegetables shows high dose-response relationship and may be a useful biomarker for isothiocyanate exposure. Eur J Nutr 46, 377-382.

16. Nermell B, Lindberg AL, Rahman M et al. (2008) Urinary arsenic concentration adjustment factors and malnutrition. Environ Res 106, 212-218.

17. Knapp EL (1947) Factors influencing the urinary excretion of calcium. I: in normal persons. J Clin Invest 26, 182-202.

18. Sato KA, Gray RW \& Lemann J Jr (1982) Urinary excretion of 25-hydroxyvitamin $\mathrm{D}$ in health and the nephrotic syndrome. J Lab Clin Med 99, 325-330.
19. Zheng W, Chow WH, Yang G et al. (2005) The Shanghai Women's Health Study: rationale, study design, and baseline characteristics. Am J Epidemiol 162, 1123-1131.

20. Cai H, Zheng W, Xiang YB et al. (2007) Dietary patterns and their correlates among middle-aged and elderly Chinese men: a report from the Shanghai Men's Health Study. Br J Nutr 98, 1006-1013.

21. Shu XO, Yang G, Jin F et al. (2004) Validity and reproducibility of the food frequency questionnaire used in the Shanghai Women's Health Study. Eur J Clin Nutr 58, 17-23.

22. Villegas R, Yang G, Liu D et al. (2007) Validity and reproducibility of the food-frequency questionnaire used in the Shanghai Men's Health Study. BrJ Nutr 97, 993-1000.

23. Seow A, Shi CY, Chung FL et al. (1998) Urinary total isothiocyanate (ITC) in a population-based sample of middleaged and older Chinese in Singapore: relationship with dietary total ITC and glutathione $S$-transferase M1/T1/P1 genotypes. Cancer Epidemiol Biomarkers Prev 7, 775-781.

24. Jiao D, Yu MC, Hankin JH et al. (1998) Total isothiocyanate contents in cooked vegetables frequently consumed in Singapore. J Agric Food Chem 46, 1055-1058.

25. Chung FL, Jiao D, Getahun SM et al. (1998) A urinary biomarker for uptake of dietary isothiocyanates in humans. Cancer Epidemiol Biomarkers Prev 7, 103-108.

26. Slot C (1965) Plasma creatinine determination. A new and specific Jaffe reaction method. Scand J Clin Lab Invest $\mathbf{1 7}$, 381-387.

27. Moore LE, Huang WY, Chatterjee N et al. (2005) GSTM1, GSTT1, and GSTP1 polymorphisms and risk of advanced colorectal adenoma. Cancer Epidemiol Biomarkers Prev 14, 1823-1827.

28. Fowke JH, Hebert JR \& Fahey JW (2002) Urinary excretion of dithiocarbamates and self-reported Cruciferous vegetable intake: application of the 'method of triads' to a foodspecific biomarker. Public Health Nutr 5, 791-799.

29. Fowke JH, Shu XO, Dai Q et al. (2003) Urinary isothiocyanate excretion, brassica consumption, and gene polymorphisms among women living in Shanghai, China. Cancer Epidemiol Biomarkers Prev 12, 1536-1539.

30. Thomson CA, Newton TR, Graver EJ et al. (2007) Cruciferous vegetable intake questionnaire improves cruciferous vegetable intake estimates. J Am Diet Assoc 107, 631-643.

31. Verkerk R \& Dekker M (2004) Glucosinolates and myrosinase activity in red cabbage (Brassica oleracea L. var. Capitata f. rubra DC.) after various microwave treatments. $J$ Agric Food Chem 52, 7318-7323.

32. Vallejo F, Tomas-Barberan F \& Garcia-Viguera C (2003) Health-promoting compounds in broccoli as influenced by refrigerated transport and retail sale period. J Agric Food Chem 51, 3029-3034.

33. Steck SE, Gammon MD, Hebert JR et al. (2007) GSTM1, GSTT1, GSTP1, and GSTA1 polymorphisms and urinary isothiocyanate metabolites following broccoli consumption in humans. J Nutr 137, 904-909.

34. Fowke JH, Chung FL, Jin F et al. (2003) Urinary isothiocyanate levels, brassica, and human breast cancer. Cancer Res $\mathbf{6 3}$, 3980-3986.

35. Dyba M, Wang A, Noone AM et al. (2010) Metabolism of isothiocyanates in individuals with positive and null GSTT1 and M1 genotypes after drinking watercress juice. Clin Nutr 29, 813-818. 